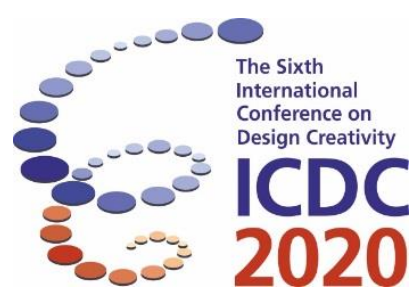

\title{
The Effect of Abstraction Methods in Bio-inspired Design - A Workshop and a Team Project Perspective
}

\author{
Lea Götz, Ida Vinkås and Helena Hashemi Farzaneh
}

Laboratory for Product Development and Lightweight Design, Technical University of Munich, Munich, Germany

\begin{abstract}
Bio-inspired design can inspire highly innovative technical solutions. However, biological inspirations in most cases have to be abstracted to transfer useful analogies to the technical domain. Is it worth the effort to use supporting methods, namely BioCards and $\mathrm{KoMBi}$, or can design teams develop bio-inspired solutions of the same quality without these methods? To answer these questions, we conducted workshops with pairs of engineering design students collaborating in an agile development project. The results of our study show that the task-specific quality of solution ideas increased significantly when using the BioCards method. The analysis of the prototypes developed throughout the project shows that the use of both abstraction methods has the highest effect on the abstraction level of analogical transfer and on the depth of understanding of the biological inspiration. These results indicate that the use of abstraction methods is recommendable for bio-inspired design teams in a comparable setting.
\end{abstract}

Keywords: biomimetics, agile product development, design-by-analogy, biocards

\section{Introduction: Abstraction in Bio-inspired Design}

Bio-inspired design - mentioning this term to anyone immediately draws attention. Most people feel intrigued by the idea of bio-inspired design; looking at nature and its solutions to solve technical challenges. Still, bio-inspiration is not a very widespread innovation method. This is reflected in the fact that very few universities offer courses in bio-inspired design.

Biological systems can rarely be used directly in the technical domain. One of the few examples is the use of purified gastric mucins as lubrification film for contact lenses (Winkeljann et al. 2017).

The direct use of biological systems in the technical domain is limited by their complexity if the scale of the system increases beyond molecular level (Sartori et al. 2010). Therefore, instead of using the biological system or imitating it by a technical solution, the essential properties of the biological system must be identified and transferred. The identification and concentration on these specific properties is called abstraction (Prechtl and Burkard 2015, p.6).

Abstraction can therefore be categorized among the activities specific to bio-inspired design (Hashemi and Lindemann 2019, p.7). Figure 1 shows further "bio-inspired design-specific" activities, such as search for biological inspiration and the transfer of bio-inspired analogies. Every bio-inspired design project requires further activities, such as the technical implementation of bio-inspired solution ideas, but they are similar to other technical projects. 


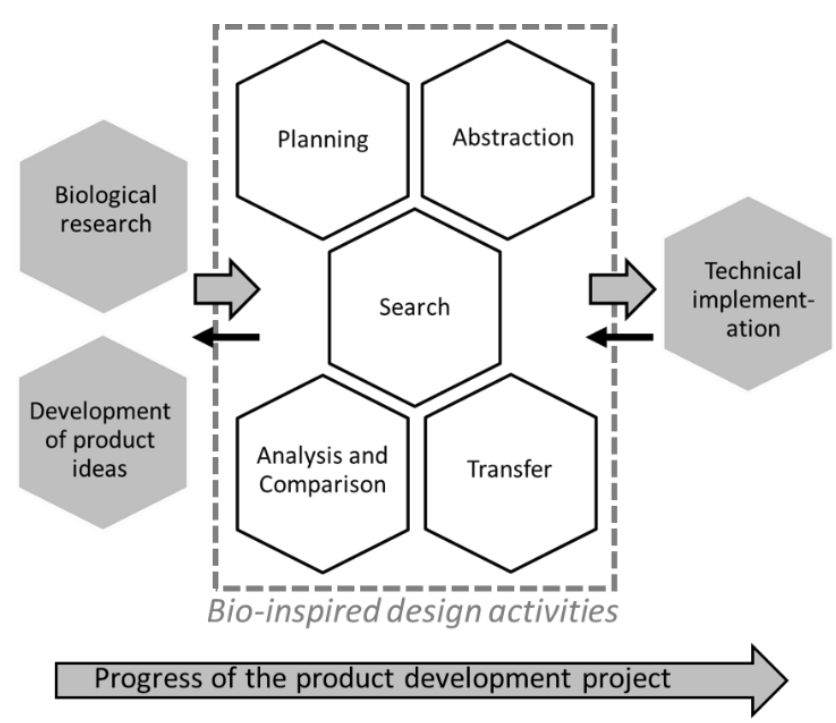

Figure 1: Bio-inspired design activities (Hashemi Farzaneh \& Lindemann 2019, p.8)

To support designers (and biologists) to abstract technical and biological systems for bio-inspired design, several abstraction methods have been developed. The majority is based on a product development perspective: they were developed based on the concept of functions as a central element.

Biocards (Lenau et al. 2011; Lenau et al. 2015) is a step-by-step abstraction approach for biological systems. Biocards use textual descriptions and sketches for describing the biological system. In creative ideation workshops, Biocards can provide simplified and, via the visualization, easily accessible information on biological systems. They can also be used in long-term collaborative research project, but one biocard cannot be used to model all aspects or functions of a biological system. Two further abstraction methods, namely SBF modeling (Goel et al. 2009; Yen et al. 2014) and SAPPhIRE (Chakrabarti et al. 2005) are more suitable for this: They provide elements and relations which are used to build up a functional model of a biological and technical system. By this means, the functions of biological and technical systems can be depicted in detail. The necessary expertise and the effort to use these abstraction methods make them less useful for creative ideation with inexperienced users. In terms of level of detail, a fourth method, KoMBi (Hashemi Farzaneh et al. 2015) is in between the previous approaches. In contrast to the previously discussed methods, KoMBi is based on aspects from both technical and biological models.

\section{Research Approach: Workshop and Project Perspective}

The aim of this work is to study the effect of using two of the abstraction methods for bio-inspired design in a bio-inspired design project: Is it worth the effort or can design teams develop bio-inspired solutions of the same quality without these methods?

For this study, BioCards were chosen as a comparably "simple" method and KoMBi was chosen as a more difficult method which allows to model a biological or technical system in more detail.

\subsection{Data collection}

The methods are studied on a student team that developed a concept and prototypes for a robotic skin in an agile project setting. The goal of the project was to develop a flexible skin for robots to be used on joints, e.g. the "elbow" joints of humanoid robots. At the same time, the robotic skin should have sensory functions to detect collisions. The project duration was 3.5 months. The student team consisted of six members: four bachelor and two master students. All of them studied mechanical engineering, engineering sciences and industrial design. Figure 2 shows the project outline. 


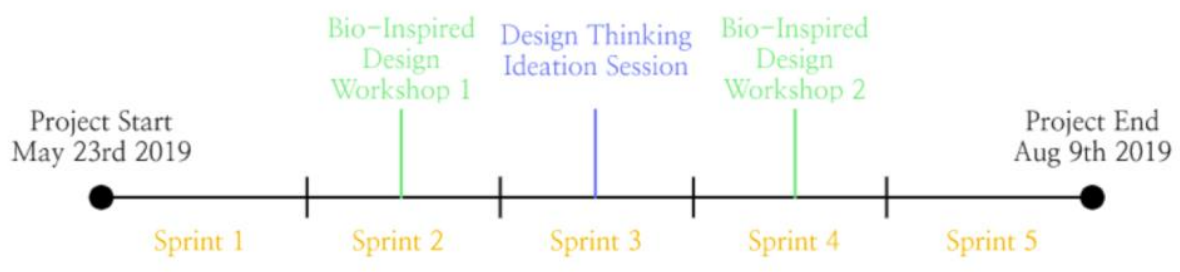

Figure 2: Project outline

The project was conducted in five agile sprints: The first two sprints mainly focussed on searching for biological and technical inspiration and on developing preliminary solution ideas. In the three following sprints, the students further detailed solution ideas and build more than 60 prototypes.

To apply the abstraction methods, two bio-inspired design workshops were conducted:

In the first workshop (sprint 2), four of the team members worked in pairs. Each pair used the abstraction method BioCards (45 minutes) on one or two biological systems and used no method (45 minutes) to explore further biological systems. One pair started with BioCards, the other pair first used no method. In both 45-minutes time slots, the pairs generated bio-inspired solution ideas based on the generated BioCards or on the other explored biological systems. This workshop allows to compare the effect of BioCards to using no method on the generation of solution ideas.

In the second workshop (sprint 4), the same procedure was used to compare the BioCards and the KoMBi method: Each pair used BioCards (45 minutes) on one or two biological systems and KoMBi (45 minutes) on one or two systems. One pair started with BioCards, the other pair first used KoMBi. Again, the pairs generated solution ideas based on the abstracted biological systems.

The difference between the two workshops is the level of detail and understanding of the explored biological systems and of the technical prototypes - before the second workshop the team had built a number of prototypes. This is the reason for choosing this sequence of using the methods: In the first workshop when the team had little knowledge on the technical problem and bio-inspired solutions, BioCards was studied as a "simple" method. In the second workshop, when the team had gained experience, $\mathrm{KoMBi}$ was studied as a more comprehensive method that allows to model more details in comparison to BioCards.

\subsection{Data analysis}

The data, i.e. the generated bio-inspired solutions were analysed on two levels:

1. The workshop level: bio-inspired solution ideas

2. The project level: bio-inspired prototypes

The aim of this procedure is to explore both the immediate effect of the use of the methods in the relatively controlled context of a workshop and the long-term effect on the prototypes built throughout the project.

The data analysis was conducted with the following procedure: The solution ideas and prototypes were first analysed by one of the authors. Subsequently another author analysed a random sample. The two authors compared and discussed their results to improve the consistency of the data analysis.

\subsubsection{Analysis of bio-inspired solution ideas (workshop level)}

To analyse the bio-inspired solution ideas, all generated solution ideas from the design experiments are identified and collected. For this work, only the ideas documented by the participants during the workshops have been taken into account. The considered ideas were documented as a note or sketch on a piece of paper or a white board.

The solution ideas were regarded on four different levels of abstraction (based on Ponn and Lindemann, 2011, p.427-460), in terms of function, physical effect, working principle and embodiment, similar to the results of previous work (Hashemi Farzaneh 2020). As an indicator for the innovative potential, both novelty and quality of the solution ideas were analysed. Assessing quality 
can be divided into a general feasibility criterion and task-specific quality criteria (procedure based on Hashemi Farzaneh 2020). Novelty and quality are common measures for creativity and have been used by a number of researchers, e.g. by Shah and Vargas-Hernandez (2003).

\subsubsection{Analysis of bio-inspired prototypes (project level)}

The level of abstraction level of all prototypes is analysed in comparison with the biological inspiration:

- Low (1): Similar geometric or material properties, however an incorrect implementation of the physical effect of the biological inspiration. The elements are transferred on embodiment level.

- Middle (2): An implementation of a physical effect similar to the biological inspiration, elements transferred on a working principle level.

- High (3): Only one orseveral functions of a biological system are transferred.

In addition, the depth of understanding of the biological inspiration is deduced from regarding all collected information on the biological system and the rationale of the developed prototypes:

- Low (1): Requirements and functions understood.

- Middle (2): Requirements, functions and working principle understood.

- High (3): Requirements, functions, working principle, and embodiment understood.

\subsubsection{Exemplary analysis of a bio-inspired solution idea and prototype}

The biological system "tortoise" was regarded in the second workshop. Figure 3 shows the BioCard and the solution idea drawn by one pair from the project group. Figure 4 displays the KoMBi model of a tortoise that was generated by the other pair. In this case the KoMBi model did not inspire a solution idea. The solution idea shown in Figure 3 was further developed into the prototype shown in Figure 5. It as to be noted that the prototype itself does not include sensors, because these were integrated into other prototypes. However, the polygons have space for the integration of sensors.
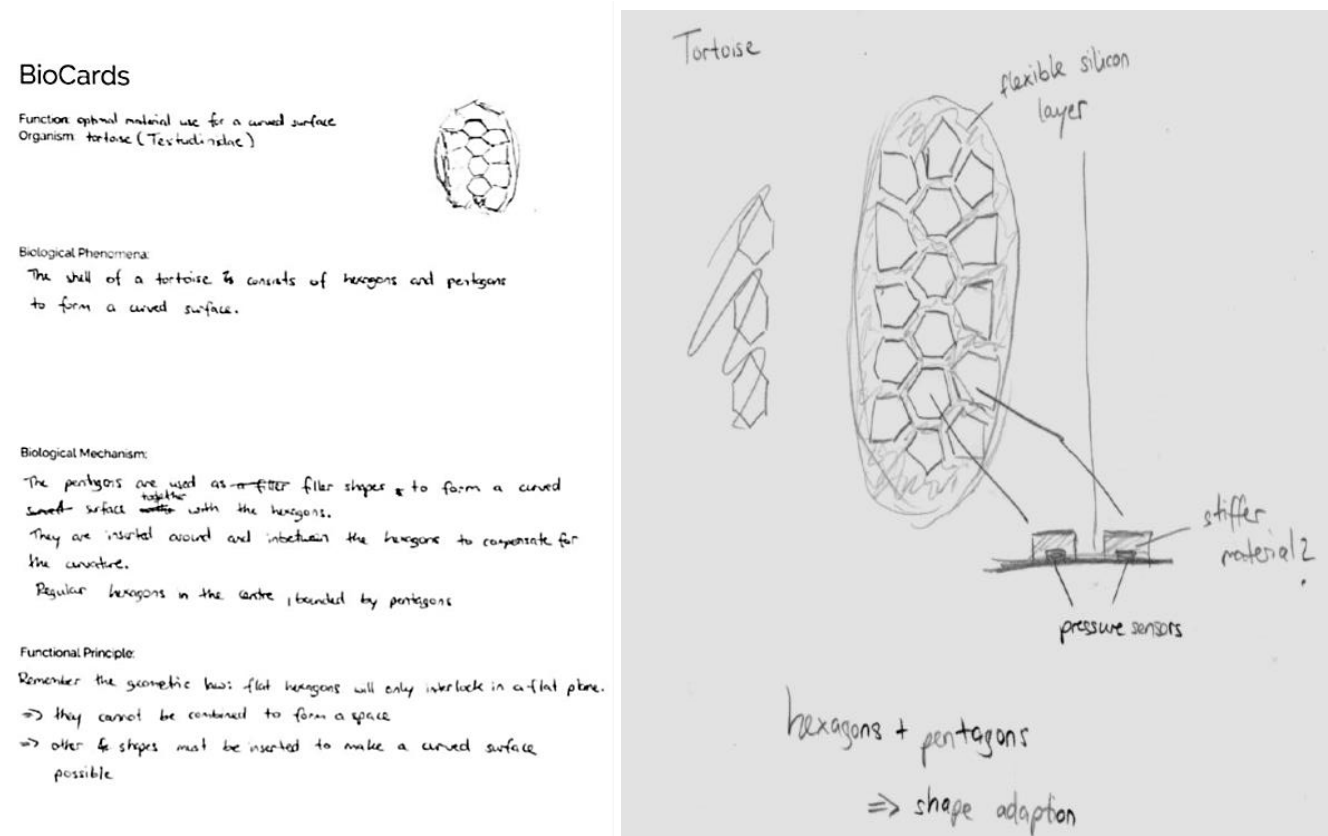

Figure 3: BioCard of a tortoise (left) and developed solution idea (right) 

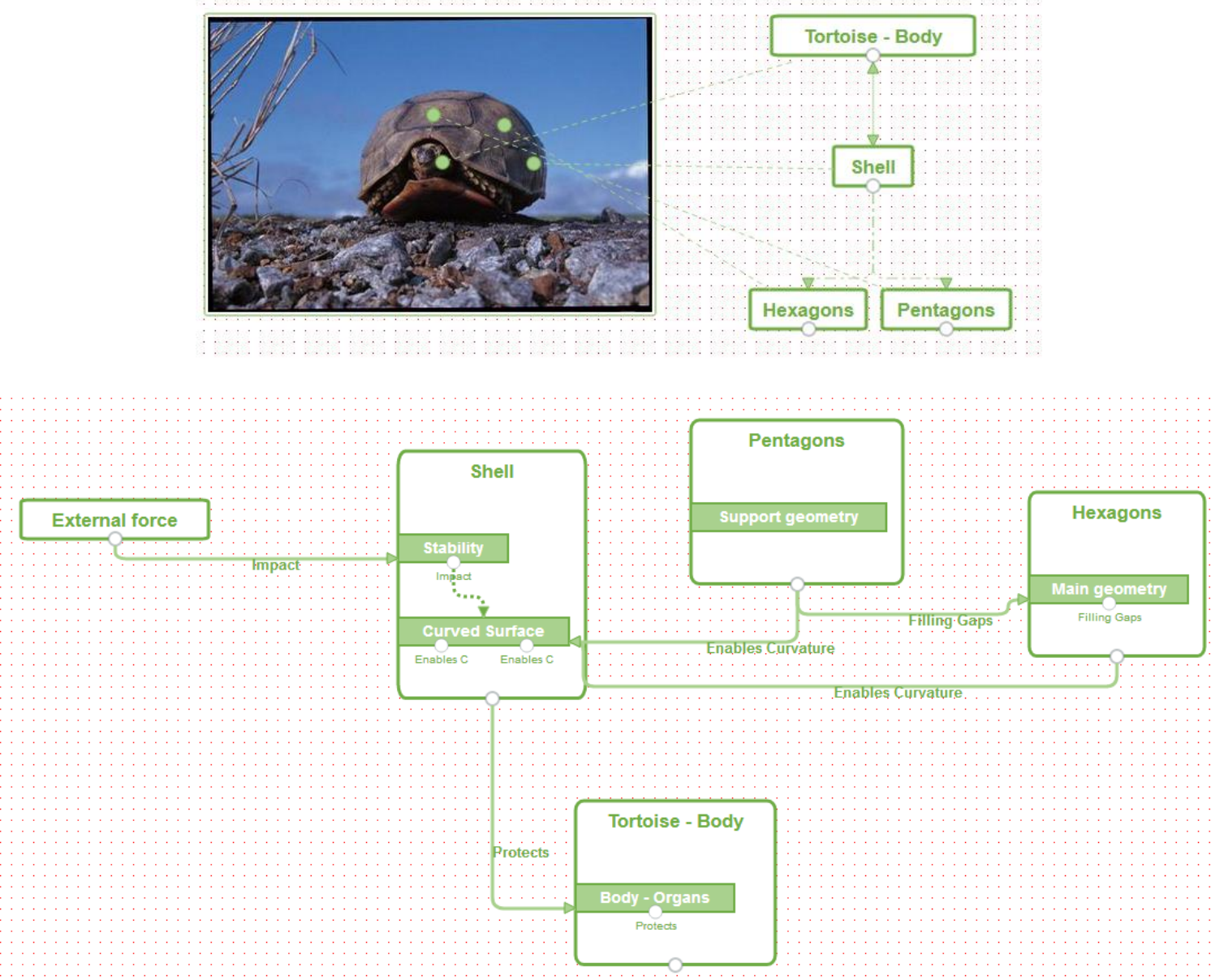

Figure 4: KoMBi model of a tortoise: System description (above) and system behaviour and properties (below)
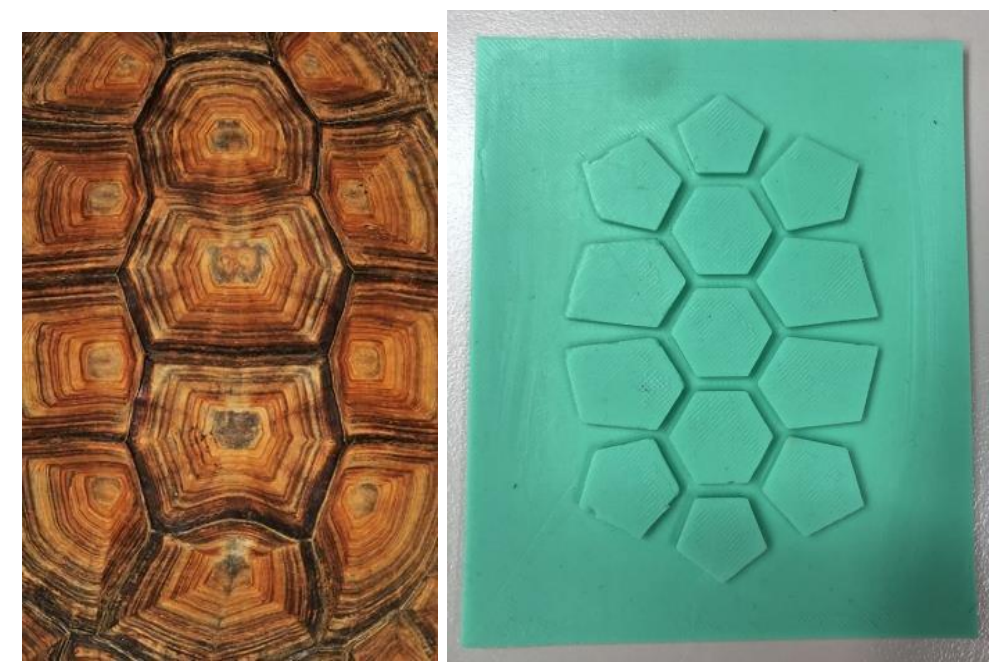

Figure 5: Detail of a tortoise shell and the developed prototype "polygons"

On workshop level, the novelty of the developed solution idea was evaluated as $66 \%$. The feasibility was evaluated as $66 \%$ and the task-specific quality as $56 \%$.

On project level, the level of abstraction of the developed prototype "polygons" was evaluated as high (3), as well as the depth of understanding of the biological system tortoise. 


\section{Results I: Analysis of Workshop Results}

Table 1 and Table 2 show the evaluation of the criteria novelty, feasibility and task-specific quality for the two workshops.

In Workshop 1, the use of BioCards resulted in 5 concepts including 12 solution ideas on working principle level. The use of "no-method" resulted in 15 concepts including 18 solution ideas on working principle level. Table 1 shows that the evaluation of all three criteria is on average higher for BioCards than for the use of "no-method".

Table 1: Comparison of BioCards and "No-method" in workshop 1

\begin{tabular}{|c|c|c|c|}
\hline \multirow{3}{*}{ Novelty } & & BioCards & No-Method \\
\hline \multirow{4}{*}{ Feasibility } & Max. rating & 3.0 & 3.0 \\
\cline { 2 - 4 } & Average rating & 2.6 & 1.93 \\
\cline { 2 - 4 } & Average rating (\%) & $86.67 \%$ & $64.33 \%$ \\
\cline { 2 - 4 } & Max. rating & 3.0 & 3.0 \\
\cline { 2 - 4 } & Average rating & 2.0 & 1.80 \\
\hline \multirow{2}{*}{$\begin{array}{c}\text { Task-specific } \\
\text { criteria fulfilment }\end{array}$} & Average rating (\%) & $66.67 \%$ & $60.00 \%$ \\
\hline
\end{tabular}

In Workshop 2, the use of BioCards resulted in 8 concepts including 15 solution ideas on working principle level, KoMBi resulted in 8 concepts including 13 solution ideas on working principle level. Table 1 shows that the evaluation of all three criteria is on average higher for BioCards.

Table 2: Comparison of BioCards and KoMBi in workshop 2

\begin{tabular}{|c|c|c|c|}
\hline \multirow{2}{*}{ Novelty } & & BioCards & KoMBi \\
\hline \multirow{4}{*}{ Feasibility } & Max. rating & 3.0 & 3.0 \\
\cline { 2 - 4 } & Average rating & 2.38 & 1.75 \\
\cline { 2 - 4 } & Average rating (\%) & $79.17 \%$ & $58.33 \%$ \\
\cline { 2 - 4 } & Max. rating & 3.0 & 3.0 \\
\cline { 2 - 4 } & Average rating & 1.88 & 2.25 \\
\cline { 2 - 4 } $\begin{array}{c}\text { Task-specific } \\
\text { criteria fulfilment }\end{array}$ & Average rating (\%) & $62.50 \%$ & $75.00 \%$ \\
\hline
\end{tabular}

The results are tested for statistical significance using the Wilcoxon-Rank-Sum test. It is a nonparametric test which is comparable to the parametric t-test, but suitable for small samples. It has been used by Lenau et al. (2015) and in previous work for this purpose. The Wilcoxon-Rank-Sum test shows statistical significance for the comparison of the task-specific quality of solution ideas based on BioCards and "no-method". Figure 6 summarizes the workshop results.

\section{Results II: Analysis of Project}

Table 3 shows the analysis of the abstraction level of the bio-inspired prototypes (A.L.) and the depth of understanding of the biological inspirations of these prototypes (U.D.).

Based on the results shown in Table 3, average values for the use of methods were calculated. The average level of abstraction of prototypes was relatively low throughout the project, with an average of 1.38. The use of BioCards increased the average level of abstraction to 1.63 and the use of KoMBi increased the level of abstraction to 1.57. However, the highest increase was achieved when both methods were used. This resulted in an average level of abstraction of 1.80 which equals an increase of over 30 percent. 


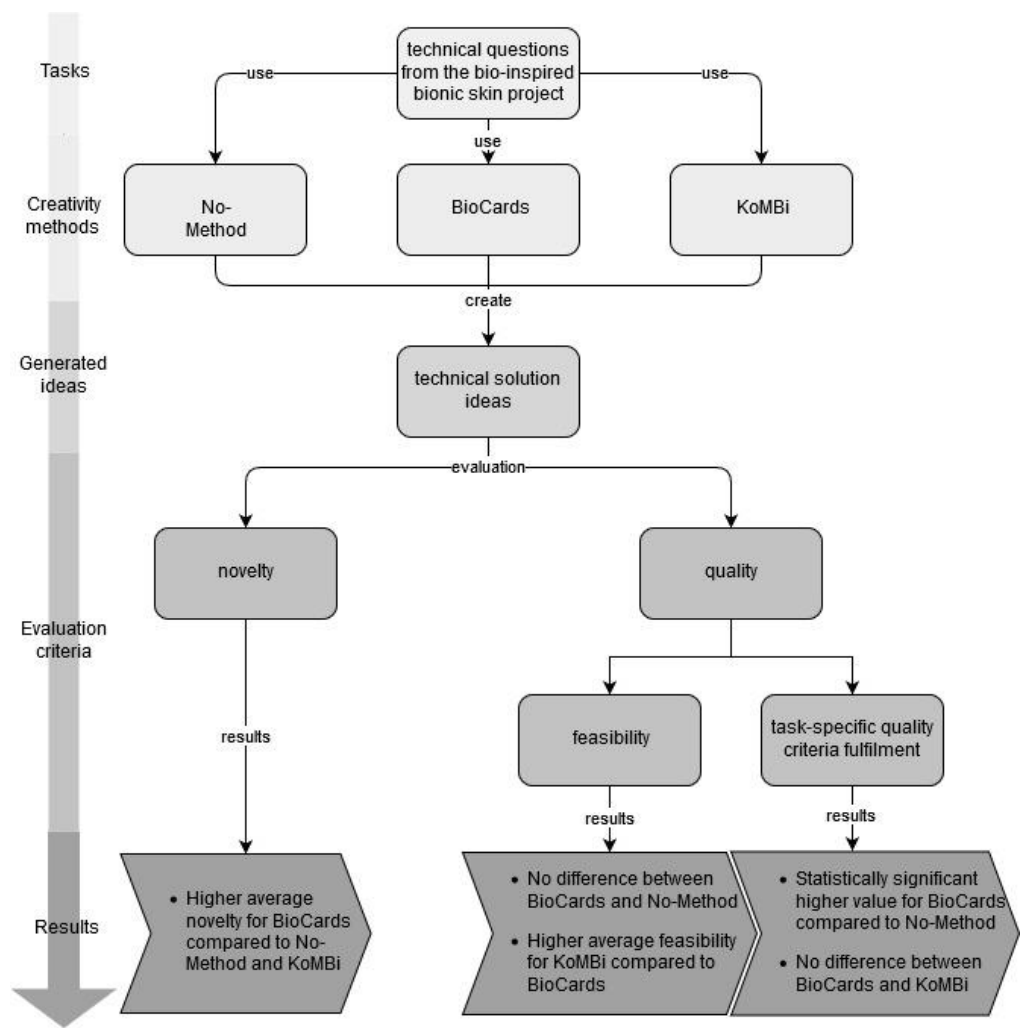

Figure 6: Summary of the analysis on workshop-level

The average depth of understanding of all concepts was 1.92. Using either BioCards or KoMBi had a similar effect on the depth of understanding, increasing the depth of understanding to 2.25 (BioCards) or 2.26 (KoMBi). As for level of abstraction, the highest increase in understanding could be noted for biological systems that had been abstracted using both methods. This increased the depth of understanding to 2.60 , an increase of over 35 percent.

Table 3: Evaluation of abstraction level (A.L.) and depth of understanding of the biological inspiration (U.D.) (Note: throughout the project brainstorming and method 6-3-5 were used as additional creativity methods to further develop concepts)

$\begin{array}{lllllll} & \text { BioCards } & \text { KoMBi } & \text { Brainst. } & \text { 6-3-5 } & \text { A.L. } & \text { U.D. } \\ \text { Scales } & \text { X } & & \text { X } & \text { X } & 1 & 2 \\ \text { Creases } & & \text { X } & \text { X } & & 1 & 1 \\ \text { Arterial Walls } & \text { X } & \text { X } & \text { X } & & 1 & 3 \\ \text { Shark Skin } & & & \text { X } & & 1 & 1 \\ \text { Hair } & \text { X } & \text { X } & \text { X } & \text { X } & 2 & 3 \\ \text { Thermal Sensing } & \text { X } & & & & 1 & 1 \\ \text { Optical Sensing } & & & \text { X } & \text { X } & 1 & 2 \\ \text { Veins \& Liquid } & & & \text { X } & \text { X } & 1 & 1 \\ \text { Snake \& Polygons } & \text { X } & \text { X } & \text { X } & \text { X } & 3 & 3 \\ \text { Spider Web \& Rings } & \text { X } & \text { X } & & & 2 & 2 \\ \text { Flower Basket } & & \text { X } & & & 1 & 2 \\ \text { Snail \& Layers } & \text { X } & & & & 2 & 2 \\ \text { Armadillo } & \text { X } & \text { X } & \text { X } & & 1 & 2\end{array}$

\section{Conclusion and Discussion}

To conclude, this work addresses the effect of abstraction methods for bio-inspired design on the development of solution ideas and prototypes: Is it worth the effort or can design teams develop bioinspired solutions of the same quality without these methods?

Two abstraction methods were chosen, BioCards as a comparably "simple" method and KoMBi as a more difficult method which allows to model a biological or technical system in more detail.

The results show that on the level of solution ideas developed immediately after applying the abstraction methods in pairs, the task-specific quality of solution ideas increased significantly when using the BioCards method. The solution ideas were then further developed into prototypes in an agile development project. The analysis of the prototypes shows that on average both methods have a 
positive effect on the abstraction level of analogical transfer and on the depth of understanding of the biological inspiration. The highest effect is achieved if both methods were applied on the biological inspiration. These results indicate that the use of abstraction methods is recommendable for bioinspired design teams in a comparable setting.

\section{References}

Chakrabarti A, Sarkar P, Leelavathamma B, Nataraju BS (2005) A functional representation for aiding biomimetic and artificial inspiration of new ideas. Artificial Intelligence for Engineering Design, Analysis and Manufacturing (AIEDAM) 19:113-132

Goel A, Rugaber S, Vattam s (2009) Structure, behavior, and function of complex systems: The structure, behavior, and function modeling language. Artificial Intelligence for Engineering Design, Analysis and Manufacturing 23:23-35

Hashemi Farzaneh H, Helms K, Lindemann U (2015) Visual representations as a bridge for engineers and biologistsin bio-inspired design collaborations. In: Weber C, Husung S, Cantamessa M, Cascini G, Marjanovic D, Srinivasan V (eds) Proceedings of the 20th International Conference on Engineering Design, Milan, Vol 2: Design Theory and Research Methodology Design Processes. Design Society, Glasgow, UK, pp 215-224

Hashemi Farzaneh H, Lindemann U (2019) A practical guide to bio-inspired design, Springer, Berlin.

Hashemi Farzaneh H (2020) Bio-inspired design: The impact of collaboration between engineers and biologists on analogical transfer and ideation. Res Eng Design (2020). https://doi.org/10.1007/s00163-020-00333-w

Lenau T, Helten K, Hepperle C, Schenkl S, Lindemann U (2011) Reducing consequences of car collsion using inspiration from nature. In: Roozenburg N, Chen LL, Stappers PJ (eds) Proceedings of the 4th World Conference on Design Research (IASDR), Delft

Lenau T, Keshwani S, Chakrabarti A, Ahmed-Kristensen S (2015) Biocards and level of abstraction. In: Weber C, Husung S, Cantamessa M, Cascini G, Marjanovic D, Srinivasan V (eds) Proceedings of the 20th International Conference on Engineering Design, Milan, Vol 2: Design Theory and Research Methodology Design Processes. Design Society, Glasgow, UK, pp 177-186

Ponn J, Lindemann U (2011) Konzeptentwicklung und Gestaltung technischer Produkte: Systematisch von Anforderungen zu Konzepten und Gestaltlösungen, 2nd edn. Springer, Heidelberg

Prechtl P, Burkard F-P (eds) (2015) Metzler Lexikon Philosophie: Begriffe und Definitionen, 3rd ed. J.B. Metzler'sche Verlagsbuchhandlung und Carl Ernst Poeschel Verlag, Stuttgart

Sartori J, Pal U, Chakrabarti A (2010) A methodology for supporting "transfer" in biomimetic design. Artificial Intelligence for Engineering Design, Analysis and Manufacturing 24:483-505

Shah, Jami J, Vargas-Hernandez N (2003): Metrics for measuring ideation effectiveness. In Design Studies 24 (2), pp. 111-134.

Winkeljann B, Boettcher K, Balzer BN, Lieleg O (2017) Mucin Coatings Prevent Tissue Damage at the CorneaContact Lens Interface. Adv. Mater. Interfaces 7:1700186. doi: 10.1002/admi.201700186

Yen J, Helms M, Goel A, Tovey C, Weissburg M (2014) Adaptive evolution of teaching practices in biologically inspired design. In: Goel AK, McAdams DA, Stone RB (eds) Biologically inspired design - computational methods and tools. Springer, London, pp 153-199 